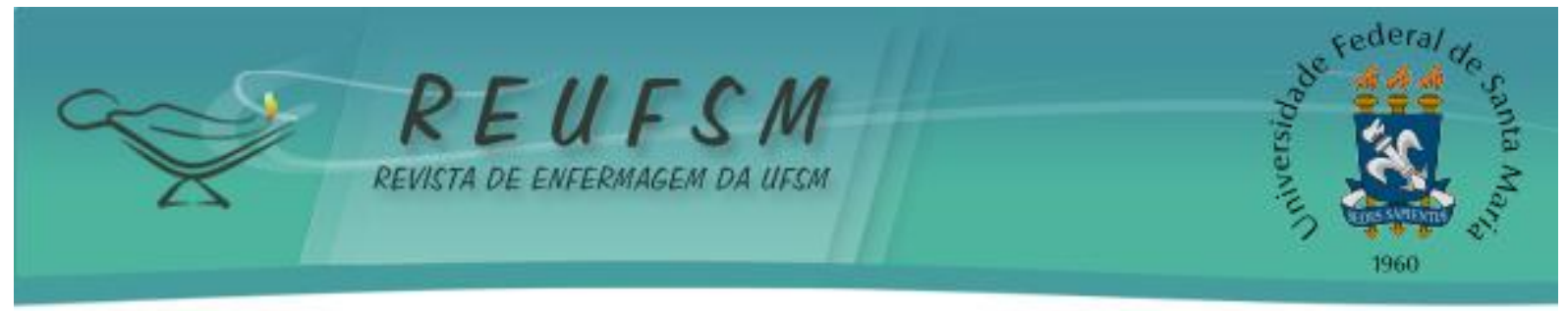

ARTIGO ORIGINAL

\title{
ATENDIMENTO DE GESTANTES HIV EM CENTRO DE TESTAGEM E ACONSELHAMENTO NA PERSPECTIVA DOS PROFISSIONAIS
}

\section{ASSISTANCE OF PREGNANT WOMEN WITH HIV CARE IN TESTING AND COUNSELING CENTERS IN THE PROFESSIONAL PERSPECTIVE}

\section{CUIDADO DE GESTANTES PORTADORAS DE HIV-SIDA EN UN CENTRO DE ASESORAMIENTO Y ORIENTACIÓN EN LA PERSPECTIVA DE LOS PROFESIONALES}

\author{
Fabiani Weiss Pereira ${ }^{1}$ \\ Michele Bulhosa de Souza ${ }^{2}$ \\ Neila Santini de Souza ${ }^{3}$ \\ Eliane Tatsch Neves ${ }^{4}$ \\ Andressa da Silveira ${ }^{5}$
}

RESUMO: Objetivo: conhecer o trabalho desenvolvido pela equipe de saúde de um Centro de Testagem e Aconselhamento (CTA) no atendimento às gestantes soropositivas para o Vírus da Imunodeficiência Humana (HIV). Método: os dados foram coletados, por meio de entrevistas semiestruturadas, com onze profissionais da saúde do CTA, em um município da região oeste do Rio Grande do Sul, em maio de 2010. Os dados foram submetidos à análise de conteúdo temática. Resultados: foram organizados em duas categorias, apontando: as dificuldades de adesão ao tratamento pelas gestantes na perspectiva dos profissionais e as estratégias de adesão ao tratamento como desafios para os profissionais. Conclusões: conclui-se a necessidade de capacitação da equipe sobre a importância de ofertar um atendimento que priorize o acolhimento. Recomenda-se que todos os profissionais da saúde do CTA ofereçam um acompanhamento que priorize e considere as necessidades e singularidades apresentadas por cada gestante.

Descritores: Enfermagem; HIV; Gestantes; Pessoal de saúde.

ABSTRACT: Objective: this is a qualitative research that aimed to know the work developed by health professional of testing and counseling centers for AIDS (TCC) in the assistance to pregnant women with Human Immunodeficiency Virus HIVIAIDS. Method: data were collected by semi structured interviews with 11 health professional of CTC in the city of the southern Brazil in May 2010. Data were submitted to content thematic analyses. Results: it was organized in two categories: the difficulties of adhesion to treatment by pregnant women in the professional perspective, and the strategies of adhesion to treatment as challenges to professionals. Conclusion: in conclusion the training of professional is necessary in order to provide assistance that priory the receptiveness. We recommend that the entire professional of TCC offered a following that consider the necessities and singularities of each woman.

Descriptors: Nursing; HIV; Pregnant women; Health personnel.

\footnotetext{
${ }^{1}$ Enfermeira. Professora Temporária da Universidade Federal do Pampa (UNIPAMPA). Especializanda em Saúde Pública com ênfase em Saúde da Família (UNINTER-EAD) e Enfermagem na Saúde da Mulher (UNIPAMPA). Email: fabianiweiss@hotmail.com

${ }^{2}$ Enfermeira. Mestre em Enfermagem. Professora Assistente do Curso de Enfermagem da Universidade Federal do Pampa (UNIPAMPA). E-mail: michelebulhosa@yahoo.com.br

${ }^{3}$ Enfermeira. Doutoranda do Dinter Enfermagem UNIFESP/EEAN/UFSM. Professora Assistente do Curso de Enfermagem da Universidade Federal do Pampa (UNIPAMPA). E-mail: neilasantini@hotmail.com

${ }^{4}$ Enfermeira. Doutora em Enfermagem. Professor Adjunto do Curso de Enfermagem e do Programa de Pós Graduação em Enfermagem da Universidade Federal de Santa Maria (UFSM). E-mail: elianeves03@gmail.com

${ }^{5}$ Enfermeira. Mestre em Enfermagem. Professora Assistente do Curso de Enfermagem da Universidade Federal do Pampa (UNIPAMPA). E-mail: andressadasilveira@gmail.com
} 


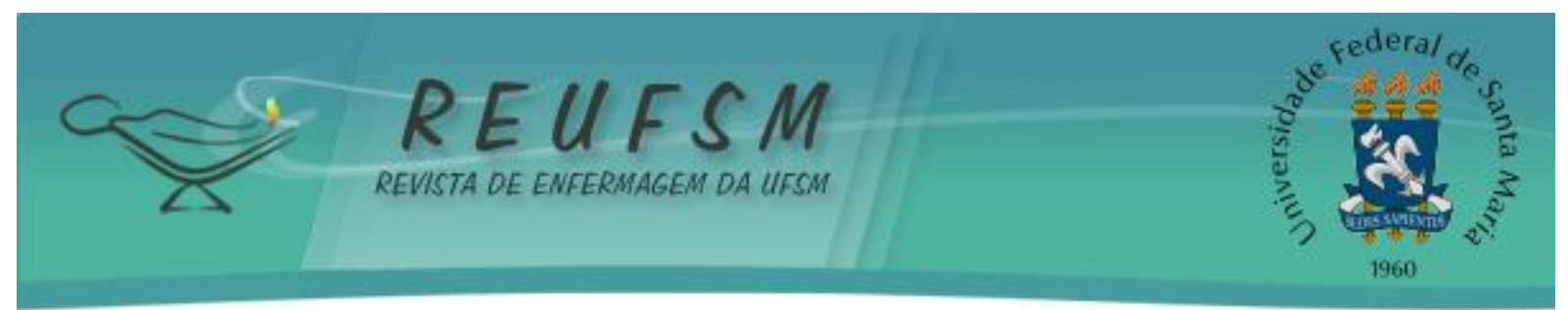

RESUMEN: Objetivo: conocer el trabajo desarrollado por el equipo de salud de un Centro de Asesoramiento y Orientaciones que asiste a gestantes seropositivas al Vírus da Inmunodeficiencia humana (VIH). Método: se recolectaron datos a través de entrevistas semiestructuradas con once profesionales de la salud del Centro en una ciudad de la región Oeste de Rio Grande do Sul en mayo de 2010. Los datos fueron sometidos a análisis de contenido temático. Resultados: fueron organizadas en dos categorías que señalaron: las dificultades de adherencia al tratamiento por parte de las gestantes desde la perspectiva de los profesionales y las estrategias de adherencia al tratamiento como desafíos a los profesionales. Conclusiones: se concluye que se trata de una necesidad de capacitación del equipo acerca de la importancia del acogimiento. Se recomienda que todos los profesionales del Centro ofrezcan un acompañamiento que priorice y considere las necesidades y singularidades presentadas por cada gestante.

Descriptores: Enfermería; VIH; Gestantes; Personal de salud.

\section{INTRODUÇÃO}

A Síndrome da Imunodeficiência Adquirida (AIDS) é a doença infecciosa de maior mortalidade no mundo. Aproximadamente 40 milhões de pessoas foram infectadas, sendo que 20 milhões já morreram desde o início da epidemia. ${ }^{1}$ Com a evolução do tratamento, a sobrevida dos pacientes aumentou e a AIDS passou a ser considerada uma doença crônica.

Dados epidemiológicos mostram que, em relação à faixa etária, dos 25 aos 49 anos, tem-se $78 \%$ de homens infectados e $71 \%$ de mulheres, caracterizando a feminilização da epidemia. Devido a isso, as estratégias de prevenção destinadas às mulheres têm sido, cada vez mais intensificadas. ${ }^{2-3}$

Considerando a feminilização da epidemia, o Ministério da Saúde recomenda a realização do teste anti-HIV, com aconselhamento e com consentimento, para todas as gestantes, na primeira consulta pré-natal. Determina ainda, que, sempre que possível, a sorologia para HIV seja repetida, no início do terceiro trimestre, visando à saúde maternoinfantil, com ênfase na prevenção da transmissão vertical para o recém-nascido. Quando a testagem não é realizada, durante o pré-natal, a mesma deve ocorrer, no momento do parto, por meio do teste rápido. ${ }^{4}$

Ao receber o diagnóstico de infecção pelo HIV, durante a gestação, a paciente deverá ser encaminhada para o Centro de Testagem e Aconselhamento e ao Serviço de Assistência Especializado (SAE), que fará o seu acompanhamento clínico como portadora do HIV. ${ }^{3}$

A geração de dados epidemiológicos locais sobre essa população torna-se de grande valor para o planejamento futuro da assistência às gestantes soropositivas para o HIV, a fim de minimizar o risco de transmissão vertical e reduzir os desfechos pós-natais desfavoráveis, envolvendo as crianças expostas. ${ }^{5}$

O acolhimento nas ações de cuidado, nos serviços de saúde, vem ganhando importância crescente no campo médico-sanitário. ${ }^{6}$ Essa temática está intimamente ligada à comunicação, em qualquer encontro profissional da saúde-gestante soropositiva para o HIV. Assim, o acolhimento, tão necessário nesta situação, pode se dar, por meio de um simples olhar explicativo, de um toque ou até, desde que eticamente possível, da sua inclusão nas questões que estão sendo socializadas pelos profissionais que desenvolvem o processo de cuidado.

Durante o atendimento, os profissionais da saúde precisam tentar compreender o que a gestante HIV positiva sente, procurando ajudá-la em seus anseios ${ }^{8}$, principalmente, quando relacionam a AIDS à morte. Mesmo que tenha ocorrido a evolução no tratamento da AIDS, "que aumentou a sobrevida do paciente e a transformou em uma doença crônica, nas representações populares a associação entre AIDS e morte é muito presente". 9:617 


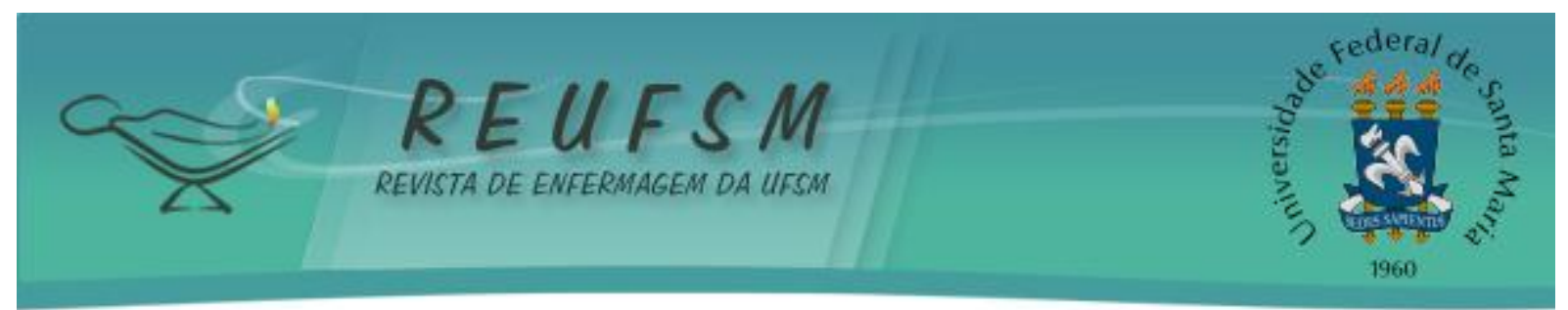

Para garantir o acesso dessa gestante aos serviços e profissionais de saúde, as consultas de pré-natal precisam seguir uma agenda flexível, para que as gestantes que apresentam efeitos colaterais ao uso de anti-retrovirais ou outra dificuldade de adesão não abandonem o tratamento. ${ }^{10}$ Percebe-se que a adesão ao tratamento com anti-retrovirais, por parte das gestantes, pode ser influenciado por alguns fatores, entre eles, destacam-se: o uso correto dos medicamentos, enfrentamento dos efeitos colaterais e, até, a ocultação dos remédios dos vizinhos, o risco de discriminação, o pacto de silêncio e o segredo do diagnóstico na família. ${ }^{10-11}$

Portanto, verifica-se o quanto é importante a presença de uma equipe multiprofissional no acompanhamento de gestantes portadoras do vírus HIV que vivenciam o tratamento para a profilaxia da transmissão vertical, oferecendo a estas mulheres uma assistência humanizada.

O acompanhamento no tratamento busca, além da prevenção da mortalidade materna e fetal, assegurar o bem estar, favorecer a compreensão e adaptação às novas vivências decorrentes da gestação, além de instrumentalizar as gestantes, em relação aos cuidados, neste período. ${ }^{8}$

Desse modo, devido à complexidade do atendimento às gestantes HIV positivas, questionou-se: como vem se desenvolvendo o atendimento a essas gestantes no CTA, na perspectiva da equipe de saúde? Assim, definiu-se como objetivo desse estudo conhecer o trabalho desenvolvido pela equipe de saúde de um CTA no atendimento às gestantes soropositivas para o HIV.

\section{MÉTODO}

Trata-se de uma pesquisa qualitativa do tipo descritiva, realizada no CTA, de um município com uma população estimada de 137.789 habitantes, situado no oeste do Rio Grande do Sul, na fronteira com a Argentina. ${ }^{12}$ Este serviço iniciou suas atividades em $1995 .{ }^{13}$

As mulheres atendidas nesse serviço são gestantes soropositivas para o HIV com idade reprodutiva entre 15 e 35 anos, que obtiveram o diagnóstico para o HIV, durante a realização do pré-natal. Grande parte dessas mulheres são de baixa renda e residem na periferia do município.

A equipe é composta por profissionais de diversas áreas de atuação e acadêmicos de enfermagem e serviço social. A dinâmica de atendimento inclui a realização de grupo de gestantes e puérperas uma vez por semana. Às gestantes é oferecido atendimento médico, psicológico, odontológico e de enfermagem. As consultas são agendadas previamente.

Os critérios de inclusão foram: ser integrante da equipe de saúde do CTA e estar desenvolvendo suas atividades profissionais, naquele local, durante o período de coleta dos dados. Foram excluídos profissionais que estavam cumprindo qualquer tipo de licença empregatícia, no período de coleta de dados. Em todos os casos, a voluntariedade dos sujeitos foi respeitada, sendo que apenas um profissional recusou-se a participar do estudo.

Para a coleta dos dados, foram realizadas entrevistas semiestruturadas, em maio de 2010, com doze profissionais de saúde elegíveis para o estudo, entre eles: dois médicos, dois enfermeiros, duas psicólogas, um dentista, três técnicas de enfermagem, uma farmacêutica e uma estagiária em serviço social. O entrevistador contatou todos os profissionais no seu local de trabalho, sendo o horário da realização da entrevista determinado por eles, conforme lhes fosse mais conveniente. A duração de cada entrevista foi de, aproximadamente, vinte minutos. Estas foram gravadas, permitindo que os entrevistados relatassem espontaneamente as suas experiências.

A coleta dos dados baseou-se em um instrumento com perguntas abertas sobre como era a realização do atendimento pela equipe, o que o profissional considerava como fundamental para a adesão da paciente ao tratamento e que estratégias eram realizadas no serviço para garantir um trabalho eficaz. Todas as entrevistas foram transcritas e 


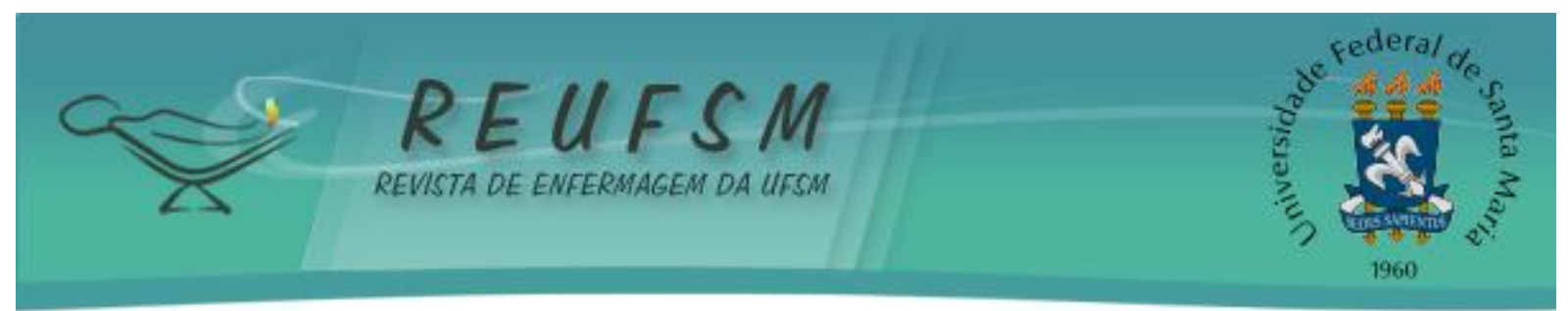

identificadas, por ordem de realização. Os entrevistados foram identificados pela letra A, seguidos de um número ordinal, em ordem crescente da realização das entrevistas ( $A 1, A 2$, A3, A4, A5, A6, A7, A8, A9, A10, A11, A12).

Os profissionais envolvidos foram convidados, sendo apresentados a cada sujeito, individualmente, o Termo de Consentimento Livre e Esclarecido, os objetivos do trabalho, a metodologia adotada, garantindo-lhes a liberdade de participação, o direito de suspender ou, até mesmo, de retirar seu consentimento e optar por deixar de participar do estudo, sem sofrer qualquer prejuízo. Assim, como thes foi assegurado o anonimato e o caráter confidencial das informações relatadas.

Para análise e interpretação dos dados, utilizou-se a análise de conteúdo temática que, "comporta um feixe de relações e pode ser graficamente apresentada através de uma palavra, de uma frase, de um resumo". ${ }^{14: 315}$ Esta análise visou a obter a sistematização e descrição do conteúdo das mensagens, tendo sido constituída de três etapas: pré-análise, exploração do material e tratamento com interpretação dos resultados obtidos.

Assim, emergiram duas categorias: as dificuldades de adesão ao tratamento pelas gestantes na perspectiva dos profissionais e as estratégias de adesão ao tratamento pelas gestantes como desafios para os profissionais.

0 projeto de pesquisa foi aprovado pelo Comitê de Ética em Pesquisa (CEP) da Universidade Federal do Pampa (UNIPAMPA), sob número de protocolo: 005.

\section{RESULTADOS E DISCUSSÃO}

Nesse artigo, são abordadas as duas categorias: as dificuldades de adesão ao tratamento pelas gestantes na perspectiva dos profissionais e as estratégias de adesão ao tratamento pelas gestantes como desafios para os profissionais.

\section{As dificuldades de adesão ao tratamento pelas gestantes na perspectiva dos profissionais}

A descoberta das gestantes do diagnóstico positivo para o HIV, durante o pré-natal, aparece como possível dificultador na adesão ao tratamento, bem como a inexistência de aconselhamento pré-teste no setor, como demonstrado nos relatos a seguir:

[...] a maioria delas recebe o resultado positivo, no momento do pré-natal. Não tem o conhecimento de serem soropositivas e, assim envolve toda uma questão emocional. (A6)

[...] qual era a minha principal preocupação? Trazer a paciente para o serviço, com isso, fazer todo um trabalho de adesão ao tratamento. (A1)

[...] agora, não estamos tendo orientação, mas, antigamente, a gente tinha. Dava orientação para vinte e poucas pessoas e elas iam para coleta. (A4)

Os profissionais preocupam-se com o que a equipe de saúde responsável pelo atendimento a essas mulheres pode ofertar, no momento em que elas recebem o diagnóstico do HIV, no pré-natal. Sendo a gravidez um momento de mudanças, relacionadas a aspectos comportamentais, físicos e emocionais, a mulher se vê diante de uma revelação, até então, desconhecida, repleta de estigmas sociais que causam os mais variados sentimentos. Assim, a equipe precisa se preocupar em estar capacitada para 


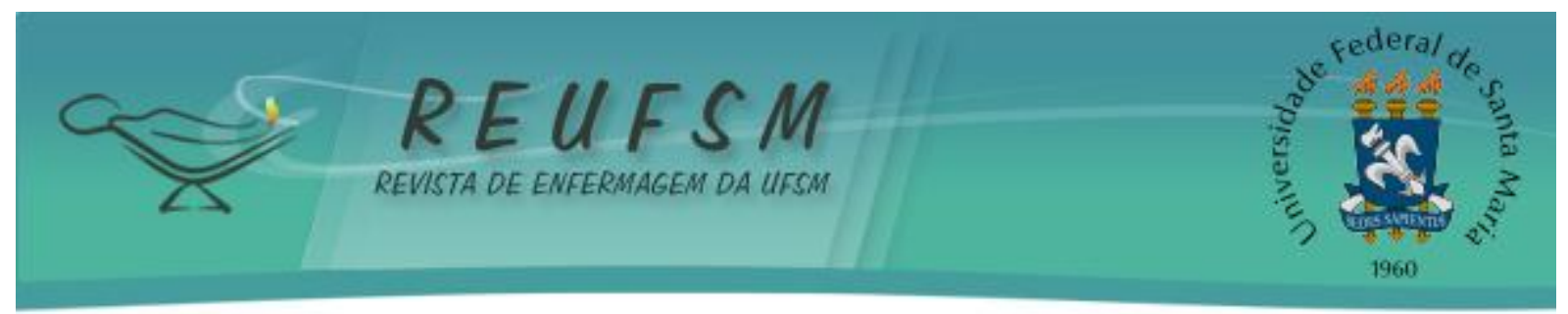

encontrar estratégias que ajudem a superar o impacto do resultado positivo para o HIV, durante a gestação, possibilitando a adesão ao pré-natal e ao tratamento.

A menção de não haver orientação no setor remete ao entendimento de que o aconselhamento pré-teste não tem ocorrido. Isto pode gerar certa dificuldade de aproximação com os profissionais da saúde, desde o momento da chegada da gestante ao setor. Este relato é preocupante, visto que essa conduta deveria estar inserida no atendimento, pois se considera que é, desde o primeiro contato com a gestante, tendo este um resultado positivo ou não, que se estabelece o primeiro momento do vínculo e troca de informações relevantes para uma melhor aproximação.

Existe o reconhecimento de ser o profissional de enfermagem aquele que pode contribuir no atendimento às gestantes soropositivas para o HIV com o aconselhamento. Assim, a enfermagem possui um papel relevante, diante de um diagnóstico sorológico de HIV positivo, durante a gestação, destacando-se a sua importância nos processos de acolhimento, vínculo e educação em saúde, que precisam estar presentes na formação do enfermeiro.

A maioria das mulheres sente orgulho pela capacidade de gerar um filho, embora os sentimentos de medos e temores façam parte da gestação. ${ }^{15}$ Entretanto, um resultado positivo para o HIV pode gerar um grande impacto, acarretando sofrimento psíquico. $3,15-16$ Assim, reconhecer-se HIV positiva na gestação pode tornar esse momento mais delicado.

Ao se deparar com a confirmação de estar soropositiva para o HIV, as mulheres apresentam reações frente a esta descoberta. Em um primeiro momento, estas reações manifestam-se sob a forma de sentimentos de desespero, atribuindo à infecção um fator determinante de sua sentença de morte, levando-as a analisar seus planos pessoais e familiares. Outro sentimento que emerge é o inconformismo e indignação, particularmente, para aquelas que não se consideravam como vulneráveis à infecção pelo vírus da Aids. ${ }^{17}$

A inserção das medicações, também, provoca insegurança, no sentido de que parece contraditória a sua ingestão, ou seja, elas precisam fazer tudo ao contrário do que sempre aprenderam. Elas precisam ingerir medicações, durante a gravidez, e prepararemse para não amamentar. Por isso, é preciso “dar suporte e ajudá-las a desconstruir conceitos há muito tempo apreendidos e apoiá-las na construção das formas de amar os filhos e no fortalecimento de vínculos afetivos com eles". ${ }^{15: 205}$

0 aconselhamento é entendido como um processo de diálogo e escuta ativa, centrado no paciente, estabelecendo confiança e resgatando os seus recursos internos, para que ele mesmo tenha possibilidade de reconhecer-se como sujeito de sua própria saúde. ${ }^{3,18-20}$ Aconselhamentos pré-teste e pós-teste são uma estratégia de acolhimento e preparo para o enfrentamento do diagnóstico. ${ }^{15,19-21} 0$ aconselhamento e o teste para mulheres grávidas é um fator-chave para a prevenção bem sucedida da transmissão vertical do HIV. ${ }^{22}$ É uma prática que pode oferecer condições indispensáveis para a interação entre as subjetividades, disponibilizando uma reflexão sobre os riscos de infecção e a necessidade de sua prevenção. ${ }^{23}$

Há mais de dez anos, o Brasil adota o acesso universal à Terapia Anti-Retroviral (TARV), sendo que, após a implementação desta política, ocorreram muitos avanços no processo saúde-doença ${ }^{24}$. Porém, "apenas o acesso universal ao tratamento não garante sua mais ampla efetividade" $25: 8$, já que promover a adesão ao tratamento vai além da simples ingestão de medicamentos. ${ }^{20}$ É preciso interação entre o paciente, a doença, o tratamento, o resultado e o serviço de saúde. ${ }^{8,26}$ Além disso, a adesão ao tratamento precisa ser entendida como um processo dinâmico, no qual o paciente e o profissional compartilham responsabilidades. ${ }^{20}$ Portanto, é necessário estabelecer uma relação de confiança entre profissional e paciente, para que haja melhor adesão ao tratamento. ${ }^{18,25-26}$

0 profissional enfermeiro, com a sua formação generalista e voltada para gerenciar o cuidado ${ }^{27}$, pode contribuir para diagnosticar as dificuldades vivenciadas pelas 


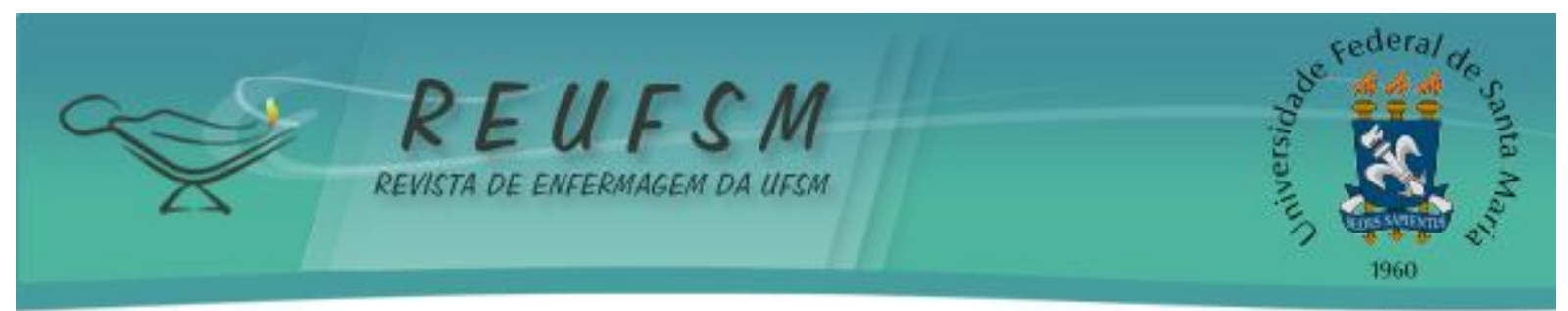

gestantes na adesão ao tratamento, porém, é preciso que toda a equipe esteja engajada nesse contexto, oferecendo um tratamento multidisciplinar e interdisciplinar. A adesão ao tratamento e ao acompanhamento em serviço de referência pelas gestantes soropositivas, associada à capacitação e sensibilização dos profissionais e gestores de saúde envolvidos na assistência a essas pacientes, possibilita uma otimização na redução da taxa de transmissão vertical a valores quase nulos. ${ }^{5}$

A negação da doença emergiu como um dificultador da adesão ao tratamento, envolvendo o preconceito e a questão da morte como uma aflição constatada nas gestantes, bem como o fator econômico, em relação à ligação do HIV à morte, como se apresenta nos relatos a seguir:

[...] a [o] HIV, às vezes, acaba associando muito com a questão da morte, de não ter futuro, não te[r] um projeto de futuro, então, a gente trabalha muito com a questão de que elas têm uma vida inteira pela frente. (A6)

[...] se tu enfrentar [es] a coisa, as pessoas não vão ter preconceito, mas tu tem [tens] que aceitar aquilo ali, se tu não aceita [s], [...] os outros não vão aceitar, entendeu [entendeste]? A primeira pessoa é a gente, o paciente tem que aceitar o que ele tem? Eu tento fazer o que eu posso, para esclarecer a doença, conscientizar. (A4)

[...] a mulher pobre, da periferia, que não tem condições e já tem um monte de filhos, ela acha que o HIV vai causar a morte dela e do filho. Ela não tem estudo, isso prejudica o tratamento. (A3)

[...] não é só, aqui, na nossa cidade, que a camada mais pobre não tem informação e associa o HIV com a morte. É em todos os lugares e isso precisa ser trabalhado. (A10)

Os profissionais destacam a associação do HIV com a morte, a não aceitação do diagnóstico e o preconceito. Relatam as dificuldades e tentam auxiliar as pacientes, em relação ao esclarecimento e aceitação, sobre sua doença e situação.

Se a não aceitação da doença pode ser uma dificuldade para a adesão da gestante soropositiva à TARV, então, ela precisa ser entendida como um processo de negociação/diálogo entre as pacientes e os profissionais de saúde, sendo que, nesse processo, são reconhecidas as responsabilidades específicas de cada um. ${ }^{26}$ Dessa forma, o profissional precisa orientar as pacientes para que alcancem o entendimento necessário. Com informação, elas poderão desenvolver potencial para identificar e tentar resolver seus problemas de saúde.

As condições socioeconômicas aparecem como dificultadoras na compreensão do HIV em associação à morte. É preciso destacar que, se o HIV pode remeter à morte, a medicação remete ao projeto de vida futuro e esta é uma decisão que precisa ser tomada pela mulher.

A tomada de decisão implica o cuidado com a saúde, de modo que haja mudança de atitude da paciente, em relação à sua doença. Para tanto, é preciso intervenção da equipe de saúde, pois não basta, apenas, aderir a esta terapia medicamentosa e utilizar o preservativo, é preciso haver um processo interno de aceitação pessoal da doença. ${ }^{28} \mathrm{~A}$ adesão à TARV é um processo dinâmico e multifatorial, abrangendo aspectos físicos, psicológicos, sociais, culturais e comportamentais ${ }^{16}$, podendo estar relacionada com a 


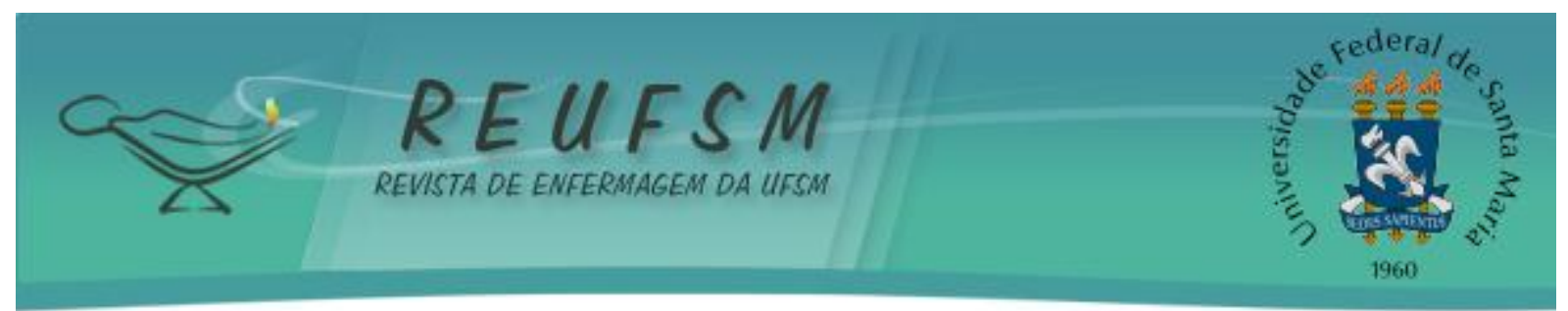

negação da doença e o autocuidado, sendo que a compreensão dos aspectos dificultadores de não adesão é o primeiro passo para o seu manejo e superação.

A vivência de dificuldades e facilidades para a adesão difere de uma pessoa a outra, pois, "é no processo de escuta que os contextos individuais específicos podem ser apropriados pela equipe, favorecendo a abordagem adequada e resolutiva". 3:20 Não basta, apenas, o profissional relatar sobre enfrentamento e aceitação da doença, é preciso agir, diante das dificuldades vivenciadas por cada gestante, tentando-se um maior vínculo e uma assistência de qualidade e, para isso, é preciso estratégias de adesão.

\section{Estratégias de adesão ao tratamento como desafios para os profissionais}

Esta categoria apresenta as estratégias utilizadas pela equipe de saúde para o atendimento às gestantes soropositivas em tratamento.

[...] acho que o acolhimento da paciente [...] e ter o serviço como referência para buscar apoio, orientação e suporte. (A1)

[...] então, a parceria com o hospital [...] é [...] importante [...] de ter uma boa comunicação, [...] por ser o único na cidade, não tem como a paciente escapar. Se eu não atendi, dentro do CTA, ela vai ser atendida no hospital. (A1)

[...] eu não sei te dizer que estratégias que cada um trabalha, dentro da sua área de atuação. (A2)

[...] acho que um controle maior, através da busca ativa, é uma estratégia para trazer a gestante. (A8)

[...] eu acredito que esse grupo que a gente iniciou, agora. E já deveria haver desde sempre, justamente, pela adesão ao tratamento. $O$ grupo de gestantes que eu faço, juntamente com as acadêmicas de enfermagem e algum convidado eventual. (A10)

Os profissionais se preocupam com a importância de possibilitar às gestantes HIV positivas um acolhimento, no sentido de que percebam que podem buscar apoio no serviço. Eles consideram o acolhimento importante e apontam o hospital como um local estratégico para a captação das gestantes, sugerindo uma possível articulação do hospital com o CTA para o seguimento do tratamento da mulher e da criança.

0 acolhimento de toda a equipe é primordial para um atendimento eficaz. A equipe precisa estar engajada no sucesso do tratamento e a participação da paciente, nesse processo, na medida em que se perceba como protagonista, podendo perceber o CTA como referência. Porém, para isso, é necessário o comprometimento de toda a equipe.

Alguns profissionais não souberam definir uma estratégia e outros mencionaram, apenas, a busca ativa e o grupo de gestantes. Neste sentido, faz-se necessário que haja desenvolvimento de estratégias para melhorar a adesão à TARV das gestantes. Devido às inúmeras causas, o enfretamento da não-adesão deve contemplar medidas com abordagens multifatoriais, pois nenhuma estratégia é eficaz sozinha. ${ }^{15}$

Com o acolhimento, podem-se criar espaços de escuta, diálogos e de recepção que proporcione a interação das pacientes e profissionais, estabelecendo um vínculo entre eles. ${ }^{3-26} 0$ acolhimento não é um espaço ou um local, não pressupõe hora ou profissional 


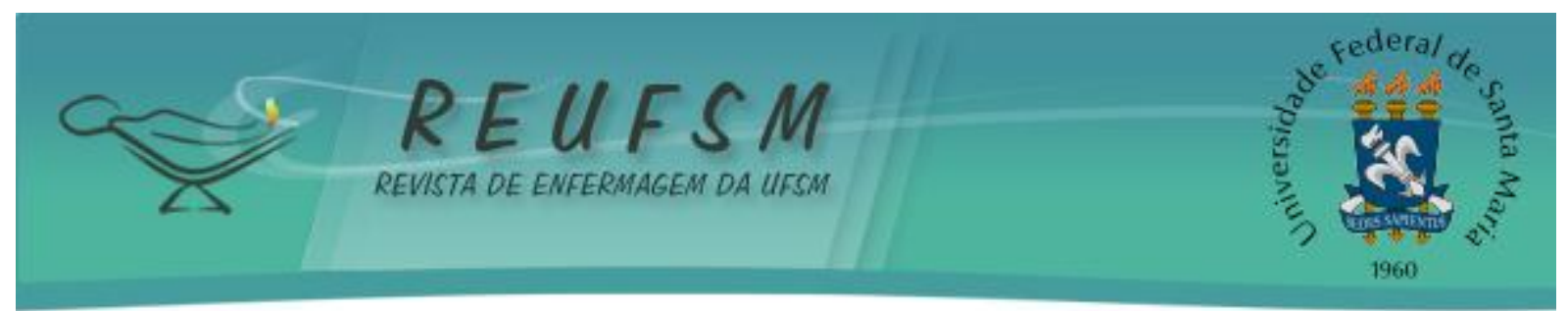

específico para fazê-lo. "Implica compartilhamento de saberes, angústias e invenções, tomando para si a responsabilidade de 'abrigar e agasalhar' outrem em suas demandas, com responsabilidade e resolutividade". $4: 9$

Salienta-se o grupo de gestantes como uma importante estratégia, pois o atendimento em grupo pode ser considerado primordial como estratégia no atendimento a essas mulheres gestantes portadoras da soropositividade para o HIV, pois este possibilita que as pacientes sintam-se acolhidas e estabeleçam vínculo com a equipe. Assim, é preciso que haja um comprometimento da equipe, em relação à continuidade do grupo, pois, em um ambiente de acolhimento, a relação entre profissional e paciente se fortalece e a intervenção técnica ocorre com maior facilidade. ${ }^{15}$ Além disso, o grupo pode tornar-se um ambiente de motivação para o tratamento, pela troca de experiências, o compartilhar de desafios e a melhor forma de superá-los. ${ }^{3}$

É importante que o serviço ofereça alternativas, de acordo com a realidade das pacientes e, para isso, é necessário "um esforço conjunto, de profissionais e pacientes, para a identificação e individualização das estratégias"15:17, pois nenhuma estratégia será eficiente, se o paciente não estiver envolvido na escolha do plano terapêutico e seguro da sua capacidade de adesão ao tratamento. 0 atendimento da equipe precisa ter como proposta utilizar o espaço aberto para abranger reflexões sobre as questões das gestantes soropositivas para o HIV, as expectativas dessas mulheres, auto-estima, preconceito, relações familiares e interpessoais, angústias, frente ao diagnóstico positivo para o HIV, e suas respectivas implicações, troca de experiências, a fala e a escuta. ${ }^{3,15}$

\section{CONCLUSÕES}

Ao conhecer o trabalho desenvolvido pela equipe de saúde de um CTA, identificou-se que estes profissionais preocupam-se com a captação e atendimento oferecido às gestantes soropositivas, bem como apontam o grupo de gestantes e o acolhimento como estratégias para adesão à TARV, porém, não especificam como estas atividades são desenvolvidas. Nesse contexto, formular estratégias que melhorem 0 atendimento, priorizem a descoberta da sorologia no pré-natal e abordem a fase de negação da doença precisam ser construídas.

Destaca-se que toda a equipe precisa estar envolvida e comprometida na construção e implementação destas estratégias, de forma interdisciplinar, para que as gestantes tenham um atendimento humanizado e qualificado. Além disso, há necessidade de capacitação das equipes que atuam em CTA sobre a importância de ofertar um atendimento que priorize o acolhimento e desenvolva estratégias de adesão ao tratamento, que envolva não só a mulher neste período, mas, também, a criança que vai nascer.

Recomenda-se que todos os profissionais da saúde do CTA ofereçam um acompanhamento que priorize e considere as necessidades e singularidades apresentadas por cada gestante soropositiva para o HIV. Ainda, que o acolhimento seja uma atitude primordial no atendimento como uma política institucional e que o grupo de gestantes seja fortalecido e inclua a participação de todos os profissionais da equipe.

\section{REFERÊNCIAS}

1. Ministério da Saúde (BR). Política Nacional de Humanização da Atenção e Gestão do SUS. O HumanizaSUS na atenção básica. Série B. Textos Básicos de Saúde. Brasília (DF): Ministério da Saúde; 2009. 


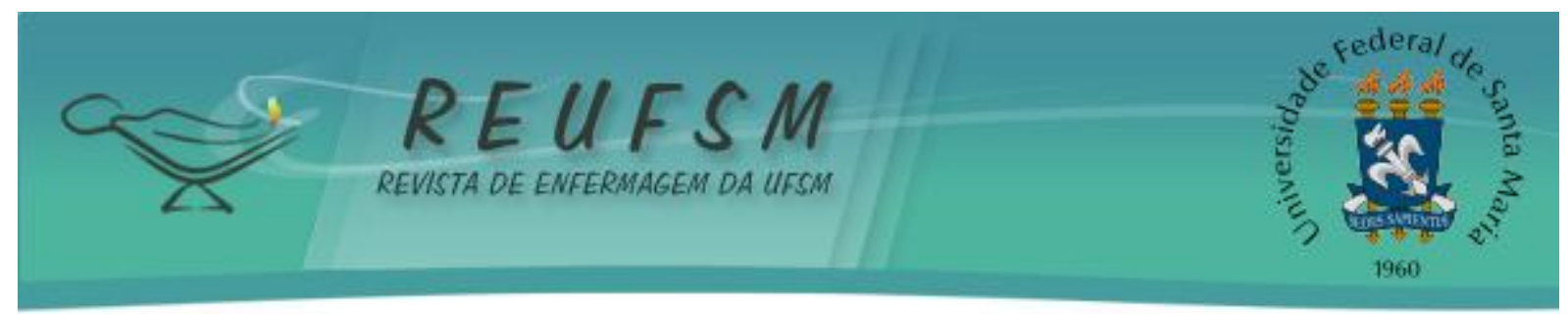

2. Schere LM, Borenstein MS, Padilha MI. Gestantes/puérperas com HIV/AIDS: conhecendo os déficits e os fatores que contribuem no engajamento para o auto-cuidado. Esc Anna Nery Rev Enferm. 2009;13(2):359-65.

3. Ministério da Saúde (BR). Secretaria de Vigilância em Saúde. Programa Nacional de DST e AIDS. Manual de adesão ao tratamento para pessoas vivendo com HIV e AIDS. Série A. Normas e manuais técnicos. Brasília (DF): Ministério da Saúde; 2008. (Série Manuais, $\mathrm{n}^{\circ} 84$ ).

4. Ministério da Saúde (BR). Secretaria de Atenção à Saúde. Núcleo Técnico da Política Nacional de Humanização. Acolhimento nas práticas de produção de saúde. Série B. Textos Básicos de Saúde. Brasília (DF): Ministério da Saúde; 2006.

5. Konopka CK, Beck ST, Wiggers D, Silva AK, Diehl FP, Santos FG. Clinical and epidemiological profile of HIV-Infected pregnant women in a service in south Brazil. Rev Bras Ginecol Obstet. 2010;32(4):184-90.

6. Teixeira RR. $O$ acolhimento num serviço de saúde entendido como uma rede de conversações. In: Roseni P, Ruben AM (organizadores). Construção da Integralidade: cotidiano, saberes e práticas em saúde. Rio de Janeiro: IMS-UERJ/ABRASCO; 2003. p.111-89.

7. Rossi FR, Lima MADS. Fundamentos para processos gerenciais na prática do cuidado. Rev Esc Enferm USP. 2005;39(4):460-8.

8. Zampieri MFM. Cuidado humanizado no pré-natal: um olhar para além das divergências e convergências[tese]. Florianópois: UFSC; 2006. 454 p.

9. Neves LAS, Gir E. Mães portadoras do HIV/AIDS: percepções acerca da severidade da infecção. Rev Esc Enferm USP. 2007;41(4):613-8.

10. Araujo MAL, Silveira CB, Melo SP. Vivências de gestantes e puérperas com o diagnóstico do HIV. Rev Bras Enferm. 2008;5(61):589-94.

11. Padoin SMM, Paula CC, Hoffmann IC, Valadão MC, Rodrigues AC, Langendorf TF. Alimentação de crianças que convivem com a aids: vivências de familiares/cuidadores em atividade grupal. Rev Enferm UFSM. [internet]. 2012 [acesso em 2012 Mai 01];2(1):213-21. Disponível em: http: / /cascavel.ufsm.br/revistas/ojs-

2.2.2/index.php/reufsm/article/view/2804/3146

12. Hentschke M, Krause AB, Magueta CMB, Braescher LM, Mattos LB, Vieira LJM, et al. Diagnóstico local de saúde: município de Uruguaiana. Porto Alegre: UFRGS, Escola de Administração; 2008.

13. Parraga APB, Rosa RS. As práticas de gestão financeira no Programa DST/AIDS de Uruguaiana (RS): reflexão sobre entraves administrativos. Rev HCPA \& Fac Med Univ Fed Rio Gd do Sul. 2005;25(1/2):68-75.

14. Minayo MCS. 0 desafio do conhecimento: pesquisa qualitativa em saúde. $10^{a}$ ed. São Paulo: Hucitec; 2007.

15. Caraciolo JMM, Shimma E. Adesão: da teoria à prática: experiências bem sucedidas no Estado de São Paulo. São Paulo: Centro de Referência e Treinamento DST/Aids; 2007.

16. Faustino QM. Intervenção cognitivo-comportamental e comportamento de adesão ao Tratamento Anti-Retroviral em pessoas vivendo com HIV/AIDS [dissertação]. Brasília: UNB; 2006. 155 p. 


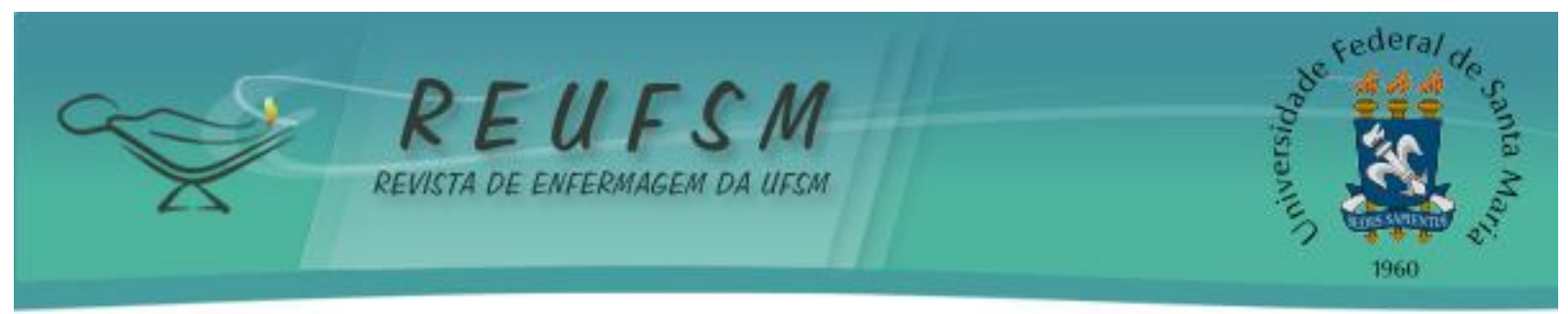

17. Moura EL, Kimura AF, Praça NS. Ser gestante soropositivo para o Vírus da Imuno deficiência Humana: uma leitura à luz do interacionismo simbólico. Acta Paul Enferm. 2010;23(2):206-11.

18. Ministério da Saúde (BR). Coordenação Nacional de DST e AIDS. Aconselhamento em DST/AIDS: princípios e diretrizes. Brasília (DF): Ministério da Saúde; 2003.

19. Ferreira MPS, Silva CMFP, Gomes MCF, Silva SMB. Testagem sorológica para o HIV e a importância dos Centros de Testagem e Aconselhamento (CTA): resultados de uma pesquisa no município do Rio de Janeiro. Ciênc Saúde Colet. 2001;6(2):481-90.

20. Braga ICC. Mulheres em idade reprodutiva infectadas pelo HIV: contribuição para a prática da enfermagem [dissertação]. Rio de Janeiro: UFRJ; 2009. 91 p.

21. Lima HMM. Adesão ao tratamento o HIV/AIDS por pacientes com AIDS, tuberculose, e usuários de droga de São Paulo [tese]. São Paulo: USP, Faculdade de Saúde Pública; 2006. 185p.

22. Hanh NTT, Gammeltoft T, Rasch V. BMC Health Services Research. Early uptake of HIV counseling and testing among pregnant women at different levels of health facilities experiences from a community based study in Northern Vietnam. BMC Health Services Research. 2011;11(29).

23. Feitosa JA, Coriolano MWL, Alencar EN, Lima LS. Aconselhamento pré-teste Anti-HIV no pré-natal. Rev Enferm UERJ. 2010;18(4):559-64.

24. Carvalho GS. Pessoas vivendo com HIV/ aids: vivencias do tratamento anti-retroviral [dissertação]. Londrina: Universidade Estadual de Londrina; 2008. 99 p.

25. Araújo MAL, Bucher JSNF, Bello PY. Eficácia do aconselhamento para doenças sexualmente transmissíveis em unidades de referência da cidade de Fortaleza. DST J Bras Doenças Sex Transm. 2004;16(1):31-7.

26. Bonolo PF, Gomes RRFM, Guimarães MDC. Adesão à terapia anti-retroviral (HIV/aids): fatores associados e medidas da adesão. Epidemiol Serv Saúde. 2007;16(4):267-78.

27. Bellato R, Pasti MJ, Takeda E. Algumas reflexões sobre o método funcional no trabalho da enfermagem. Rev Latinoam Enferm. 1997;5(1):75-81.

28. Pereira MHGG, Costa F. O autocuidado em mulheres portadoras de HIV/AIDS. Psicol Saúde Doenças. 2006;7(2):255-69.

Data de recebimento: 07/05/2012

Data de aceite: 24/06/2012

Contato com autor responsável: Fabiani Weiss Pereira

Endereço: BR 472 - Km 592 - Caixa Postal 118 - Uruguaiana/RS

CEP: $97500-970$

E-mail: fabianiweiss@hotmail.com 